\title{
Aislamiento, identificación y daños asociados al síndrome de la muerte súbita en el cultivo de soja en Argentina
}

\author{
María Mercedes Scandiani ${ }^{1,2}$, Marcelo Aníbal Carmona ${ }^{3}$, Alicia Graciela Luque ${ }^{2}$, Kedma da Silva Matos ${ }^{4}$, \\ Lisandro Lenzi ${ }^{5}$, Ángela Norma Formento ${ }^{6}$, Cristina Valeria Martinez ${ }^{7}$, Mónica Raquel Ferri ${ }^{1}$, Melina Lo \\ Piccolo $^{2}$, Mirta Tartabini ${ }^{2}$, Diego Alvarez ${ }^{8} \&$ Francisco Sautua $^{3}$
}

${ }^{1}$ Laboratorio Agrícola Río Paraná, 2930, San Pedro, Argentina; ${ }^{2}$ Centro de Referencia de Micología-CEREMIC, Facultad de Ciencias Bioquímicas y Farmacéuticas, Universidad Nacional de Rosario, Rosario, Argentina; ${ }^{3}$ Cátedra de Fitopatología, Facultad de Agronomía, Universidad de Buenos Aires, Buenos Aires, Argentina; ${ }^{4}$ Universidade Federal de Lavras, Lavras, MG, Brasil; ${ }^{5}$ INTA, EEA Marcos Juárez, Córdoba, Argentina; ${ }^{6}$ INTA, EEA Paraná, Entre Ríos, Argentina; ${ }^{7}$ EEAOC, Tucumán, Argentina; ${ }^{8}$ Lares SRL, Pergamino, Buenos Aires

Autor para correspondencia: María Mercedes Scandiani, e-mail: labagricola@sanpedro.com.ar

\section{RESUMEN}

Los objetivos de este trabajo fueron aislar e identificar los agentes causales del síndrome de la muerte súbita (SMS) en muestras provenientes de diferentes localidades de Argentina, y cuantificar la incidencia de la enfermedad y los daños causados a campo en el rendimiento. Se analizaron 215 raíces provenientes de plantas con síntoma foliares típicos de SMS para el aislamiento e identificación de los agentes causales. Para realizar las pruebas de patogenicidad se efectuaron dos bioensayos en invernáculo. Los daños fueron estimados en un ensayo a campo situado en Pergamino a través de la cuantificación del rendimiento de muestras apareadas. El 36\% (78 raíces) de las raíces presentó signos de Fusarium, compatibles con los causantes de SMS. Los estudios morfológicos realizados permitieron identificar 35 aislamientos como F. tucumaniae y 18, como F. virguliforme. Otros ocho aislamientos no pudieron identificarse. Todos los aislamientos inoculados reprodujeron los síntomas foliares típicos de SMS. Se observaron diferencias entre el peso de mil granos de plantas sanas y de plantas enfermas y entre el rendimiento potencial y el real $(p<0,05)$. Los daños promedio fueron de $1514 \mathrm{~kg} / \mathrm{ha}(\mathrm{rango} 192-3770 \mathrm{~kg} / \mathrm{ha})$. Los resultados obtenidos corroboraron la distribución y predominio de F. tucumaniae y F. viguliforme en el área estudiada y su potencial destructivo.

Palabras clave: Glycine max, Fusarium tucumaniae, Fusarium virguliforme, SMS.

\begin{abstract}
Isolation, identification and yield losses associated with sudden death syndrome in soybeans in Argentina

The main objectives of this work were to isolate and identify the causal agents of sudden death syndrome (SDS) from samples collected in different Argentinean localities, to quantify its incidence, and to estimate yield losses. Two hundred and fifteen roots from plants with typical SDS foliar symptoms were analyzed. In order to perform pathogenicity tests, two bioassays were conducted in the greenhouse. Yield losses were estimated in a field trial located in Pergamino by quantification and comparison of yield of paired samples. Thirty-six percent of total roots (78 roots) presented signs of Fusarium, compatible with signs caused by SDS. Morphological studies allowed the identification of 35 isolates as F. tucumaniae and 18 as F. virguliforme. Other eight isolates could not be identified. All isolates that were inoculated reproduced typical SDS foliar symptoms. Significant differences were observed between healthy and diseased plants for 1000-grain weight and between potential and real yield $(\mathrm{p}<0.05)$. Average yield loss was $1514 \mathrm{~kg} / \mathrm{ha}(\mathrm{range} 192-3770$ $\mathrm{kg} / \mathrm{ha}$ ). These results corroborated the distribution and predominance of $F$. tucumaniae and $F$. viguliforme in the area under study and its destructive potential.
\end{abstract}

Key words: Glycine max, Fusarium tucumaniae, Fusarium virguliforme, SDS.

El síndrome de la muerte súbita (SMS) es una importante enfermedad fúngica de la soja [Glycine max (L.) Merr.]. En la Argentina fue identificada por primera vez en la región pampeana norte, durante la campaña 1991/92, y en el noroeste argentino (NOA) en la campaña siguiente (Ploper, 1993). Progresivamente, se convirtió en una de las patologías de mayor importancia con daños en variedades muy susceptibles con un rango de $15 \%$ y $90 \%$, en el centro y norte del país, respectivamente (Ploper, 1993). Los primeros síntomas generalmente se manifiestan a partir de floración, aunque pueden observarse en etapas vegetativas, y consisten en clorosis con posterior necrosis internerval, pudiendo aparecer en estados vegetativos tempranos como V4 (Rupe \& Hartman, 1999). Frecuentemente, se produce la caída de los folíolos, quedando los pecíolos adheridos al tallo. El sistema radicular es afectado, exibiendo pigmentación rojiza en algunas ocasiones, en forma externa y basal, observándose menor desarrollo y podredumbre de raíces. Las plantas se marchitan, mueren en forma aislada, pero 
Aislamiento, identificación y daños asociados al síndrome de la muerte súbita en el cultivo de soja...

más comúnmente en grupos, o a veces distribuidas por todo el campo.

El agente causal del SMS era conocido como Fusarium solani f. sp. glycines antes de realizarse los estudios filogenéticos moleculares, los que conjuntamente con estudios morfológicos y patogénicos realizados desde 2003 demostraron que el SMS es causado por cuatro especies de Fusarium, habitantes del suelo, muy relacionadas entre sí (F. virguliforme O’Donnell \& T. Aoki, F. tucumaniae T. Aoki, O’Donnell, Yos. Homma \& Lattanzi, F. brasiliense T. Aoki \& O'Donnell, y una especie nueva: Fusarium crassistipitatum Scandiani, T. Aoki \& O'Donnell, sp. nov. (Aoki et al., 2005, 2012). Se determinó en Argentina la existencia de los cuatro agentes causales, con prevalencia de $F$. tucumaniae, seguido por $F$. virguliforme, siendo esta última la especie dominante en Estados Unidos (Scandiani et al., 2004; O’Donnell et al., 2010; Aoki et al., 2012). En Brasil, estudios efectuados con pocos aislamientos, demostraron que al menos se encuentran presentes: $F$. tucumaniae, F. brasiliense y F. crassistipitatum sp. nov. (Arruda et al., 2005; O'Donnell et al., 2010; Aoki et al., 2012). En 2006 se demostró la ocurrencia de la forma sexual (peritecios) de F. tucumaniae mediante cruzamientos en condiciones de laboratorio (Covert et al., 2007) y en 2010 se la encontró en la naturaleza (en forma sexual, cerca de Pergamino, en Fontezuela, 3355 '53.13' 'S; 60²8'11.19' 'O, WGS084) (Scandiani et al., 2011).

El objetivo del trabajo fue realizar el aislamiento, identificación y pruebas de patogenicidad de los agentes causales del SMS en muestras de raíces provenientes de diferentes localidades de la Pradera Pampena Argentina; y al mismo tiempo efectuar la cuantificación de la incidencia de la enfermedad y la estimación de los daños causados en el rendimiento de soja en un ensayo bajo condiciones de infección severa, durante la campaña 2010/2011.

Para el relevamiento, se tomaron muestras provenientes de diversas regiones donde la enfermedad es endémica. Las mismas fueron analizadas observándose 215 raíces de plantas de soja con síntoma foliares típicos de SMS. Éstas, se incubaron en cámara a $10^{\circ} \mathrm{C}$, envueltas individualmente en papel húmedo, para estimular la producción de signos de Fusarium.

Los aislamientos se obtuvieron mediante la técnica de cultivo directo de macroconidios. Los pasos seguidos para cumplir con este objetivo fueron: a) localización de signos compatibles con Fusarium causantes de SMS (colores: azul, verde, amarillo) bajo lupa estereoscópica $40 \mathrm{X}$; b) transferencia de macroconidios con aguja histológica estéril a un portaobjetos esterilizado conteniendo una gota de agua destilada estéril; c) confirmación de la presencia de macroconidios compatibles con los de las especies causantes de SMS, al microscopio 100 a 200X; d) siembra por estrías con ansa bacteriológica estéril en placas de $90 \mathrm{~mm}$ conteniendo agar papa glucosa más estreptomicina (APDS). Este procedimiento se realizó hasta el descarte de la raíz cuando se obtuvieron los cultivos o por alta contaminación; e) incubación de las placas invertidas durante 4 días a $25^{\circ} \mathrm{C}$ bajo 12 horas de luz; f) observación de las estrías y transferencia de todas las colonias sospechosas de ser Fusarium causantes de SMS (crecimiento lento), en forma individual a placas de $60 \mathrm{~mm}$ con APDS.

Una vez obtenidos los cultivos puros se realizó la identificación mediante el estudio de características morfológicas. Se seleccionaron aquellos cultivos que presentaron velocidad de crecimiento lenta (colonia menor a $2 \mathrm{~cm}$ de diámetro a los cuatro días de cultivo en las condiciones anteriormente descriptas), los que sumaron un total de 61 aislamientos. Posteriormente, se determinó la longitud y ancho promedio de los macroconidios, así como la presencia de microconidios en forma de coma (Scandiani et al., 2011).

Para realizar las pruebas de patogenicidad se realizaron dos bioensayos; en uno, se evaluaron tres aislamientos, dos identificados como $F$. tucumaniae y uno, no identificado, inoculados por el método de la capa, utilizando granos de sorgo como sustrato (Scandiani et al., 2011). Por otro lado, se evaluaron otros cuatro aislamientos de $F$. tucumaniae, inoculados mediante riego con una suspensión de $10^{5}$ conidios $/ \mathrm{mL}$, de acuerdo a lo descripto por Gongora \& Leandro (2011). Se registró la incidencia de plantas (IP) con síntomas foliares de SMS, a los 30 días mediante la fórmula IP $(\%)=$ número Pe / Tp x $100(\mathrm{Pe}=$ plantas enfermas, $\mathrm{Tp}=$ total de plantas $)$. La severidad de los síntomas foliares (\% de área foliar necrosada y clorótica) se evaluó mediante una escala de 1 a 5 , donde $1=$ planta sana y 5 = planta muerta (Aoki et al., 2005; Scandiani et al., 2011), y se determinó el peso fresco de la parte aérea según Li et al. (2009). En el primer ensayo se incluyó como testigo un aislamiento de $F$. tucumaniae, y en el segundo ensayo, uno de $F$. virguliforme. En ambos ensayos se dejaron testigos absolutos, con sustrato no infestado. Los datos obtenidos fueron analizados mediante ANOVA y la separación de las medias por el test de las Diferencias Mínimas Significativas (DMS) al 5\%.

Para estimar daños se realizó un ensayo a campo sobre un lote sembrado con la variedad de soja DM 4670 en la localidad de Fontezuela, partido de Pergamino (Buenos Aires). El sistema de producción fue el de siembra directa con antecesor maíz.

Para cuantificar la enfermedad y los daños se demarcaron sobre un lote severamente infectado, seis líneas de siembra de $10 \mathrm{~m}$ cada una constituyendo un total de seis repeticiones. Cada una de estas líneas de siembra estaba separada aproximadamente $20 \mathrm{~m}$ entre sí. La incidencia de la SMS fue determinada a través del porcentaje de plantas que presentaban síntomas foliares típicos visibles al estadio $\mathrm{R} 3$ respecto al número total de plantas. Para ello se procedió a marcar en la base del tallo con precintos azules, las plantas enfermas y con rojos, a las sanas y se calculó la incidencia con la siguiente fórmula: I P $(\%)=$ número Pe / Tp x 100 (donde: $\mathrm{Pe}=$ plantas enfermas, $\mathrm{T} p=$ total de plantas). 
Cada repetición fue cosechada separadamente, determinando el rendimiento expresado en gramos y posteriormente transformado a $\mathrm{kg} / \mathrm{ha}$ y el peso de 1000 granos. La humedad fue previamente corregida al 13\%. La cuantificación de daños fuerealizadamediantelametodología adaptada de Reis et al (1996): 1) Cálculo del rendimiento potencial (RENDPOT) que es el rendimiento que se hubiera obtenido si no se hubiese presentado la enfermedad. Para su estimación se consideró el rendimiento de las plantas sanas de cada línea y se lo ajustó de acuerdo al número total de plantas de cada línea. 2) Cálculo del rendimiento real (RENDREAL), que es el rendimiento cosechado en cada una de las líneas del ensayo. Para su estimación se sumaron el rendimiento de las plantas sanas y el rendimiento de las plantas enfermas. 3) Los daños se estimaron a través de la diferencia de RENDPOT - RENDREAL. Se aplicó el test $t$ de Student para diferencias entre muestras apareadas a un nivel de $5 \%$ de probabilidad.

El 36\% (78 raíces) de las raíces estudiadas presentó signos de Fusarium, compatibles con los producidos por las especies causantes de SMS, en la primera observación y se realizaron los aislamientos según la metodología descripta. El porcentaje de eficiencia de los aislamientos, considerando las raíces con signos en la primera observación, fue del 92\%. Cuando las raíces presentan un largo período de almacenamiento (20 días o más) entre el papel, éste se seca y aumentan los saprófitos, dificultando el aislamiento. Las raíces sin signos, si se encontraban en buen estado de conservación, se incubaron nuevamente o se descartaron cuando presentaron alto grado de descomposición.

Los aislamientos de Fusarium causantes de SMS obtenidos presentaron la siguiente distribución geográfica: $31 \%$ de Santa Fe, 33\% de Buenos Aires, 29\% de Córdoba y $7 \%$ de Entre Ríos.

Los estudios morfológicos realizados permitieron identificar 35 aislamientos como F. tucumaniae y 18 como F. virguliforme. Otros ocho aislamientos de Fusarium causantes de SMS no pudieron identificarse en base a sus caracteres micromorfológicos, lo que pone de manifiesto lo difícil que es la clasificación taxonómica de estos hongos.
En todas las provincias mencionadas anteriormente predominaron los aislamientos de $F$. tucumaniae, a excepción de Buenos Aires donde el 55\% de los mismos correspondió a $F$. virguliforme.

Las pruebas de patogenicidad permitieron comprobar que todos los aislamientos seleccionados, reprodujeron los síntomas foliares típicos de SMS por ambos métodos de inoculación. Todos los aislamientos causaron elevadas incidencias de plantas con síntomas foliares de SMS, y severidades mayores a 3, indicando que las plantas presentaban síntomas de clorosis y necrosis internerval. Todas las plantas infectadas presentaron menor desarrollo, que se vio reflejado en el peso fresco significativamente menor obtenido en las plantas inoculadas con respecto a los testigos correspondientes (Tablas 1 y 2).

La enfermedad se presentó en el lote en el cual se realizó el ensayo en forma generalizada y distribuida al azar. Por ello, el diseño establecido en líneas de siembras de $10 \mathrm{~m}$ resultó adecuado. Mediante el aislamiento de plantas enfermas, se determinó la coexistencia en el lote de las especies predominantes en Argentina, $F$. virguliforme y $F$. tucumaniae. Los patógenos aislados de las plantas enfermas correspondieron a $F$. virguliforme $(55 \%), F$. tucumaniae $(22 \%)$ y los no identificados, que correspondieron a Fusarium spp. de crecimiento lento (22\%). Como se ha mencionado en trabajos previos, la identificación en base a la morfología de los aislamientos causantes del SMS de la soja es una tarea compleja. Los conidios en forma de coma no siempre se observan en los cultivos de $F$. virguliforme. El tamaño de los macroconidios de $F$. virguliforme y de $F$. tucumaniae observados en Argentina, en algunas ocasiones, no presentan las diferencias tan notables como las descriptas en la bibliografía (Aoki et al., 2005). Además, en Argentina existen $F$. brasiliense y $F$. crassistipitatum causales también de SMS, lo que dificulta aún más la identificación por métodos clásicos.

La demarcación de las plantas enfermas versus sanas en cada una de las seis líneas de siembra, resultó sencilla por el diagnóstico visual de los síntomas foliares causados por la enfermedad. La incidencia promedio fue del $51 \%$ con

TABLA 1 - Valores de incidencia y severidad foliar del síndrome de la muerte súbita y peso fresco de la parte aérea por planta, registrados en el cv. de soja NA4613RG registrados a los 30 días después de inoculados (inóculo en grano de sorgo)

\begin{tabular}{lccc}
\hline \hline $\begin{array}{l}\text { Aislamiento } \\
\text { CCC }^{1}\end{array}$ & Incidencia (\%) & $\begin{array}{c}\text { Severidad } \\
(\mathbf{1 - 5})^{\mathbf{2}}\end{array}$ & $\begin{array}{c}\text { Peso fresco por planta } \\
\text { (parte aérea) (g) }\end{array}$ \\
\hline Fusarium tucumaniae CCC 126-02 & $100,00 \mathrm{a}$ & $3,62 \mathrm{~b}$ & $1,437 \mathrm{c}$ \\
Fusarium tucumaniae CCC 132-11 & $94,79 \mathrm{a}$ & $3,91 \mathrm{ab}$ & $0,754 \mathrm{e}$ \\
Fusarium tucumaniae CCC 130-11 & $93,42 \mathrm{ab}$ & $4,09 \mathrm{ab}$ & $0,897 \mathrm{de}$ \\
Fusarium spp. CCC 131-11 & $100,00 \mathrm{a}$ & $4,70 \mathrm{a}$ & $0,517 \mathrm{e}$ \\
Testigo & $0,00 \mathrm{c}$ & $1,00 \mathrm{c}$ & $2,988 \mathrm{ab}$ \\
\hline CV\% & 8,52 & 10,68 & 20,19 \\
\hline
\end{tabular}

Letras iguales dentro de cada columna indican diferencias no significativas según DMS 5\%.

${ }^{1}$ CCC: Colección de Cultivos del CEREMIC (Centro de Referencia de Micología), Fac. de Cs. Bioquímicas y Farmacéuticas, UNR, Rosario, Argentina.

${ }^{2}$ Escala de 1 a 5 donde 1 = planta sana, y 5 = planta muerta. 
Aislamiento, identificación y daños asociados al síndrome de la muerte súbita en el cultivo de soja...

TABLA 2 - Valores de incidencia y severidad foliar del síndrome de la muerte súbita y peso fresco de la parte aérea por planta, registrados en el cv. de soja NA4613RG registrados a los 30 días después de inoculados (suspensión conidial)

\begin{tabular}{lccc}
\hline \hline $\begin{array}{l}\text { Aislamiento } \\
\text { CCC }^{\mathbf{1}}\end{array}$ & Incidencia (\%) & $\begin{array}{c}\text { Severidad } \\
(\mathbf{1 - 5})^{\mathbf{2}}\end{array}$ & $\begin{array}{c}\text { Peso fresco por planta } \\
\text { (parte aérea) (g) }\end{array}$ \\
\hline Fusarium tucumaniae CCC 128-11 & $100,00 \mathrm{a}$ & $5,00 \mathrm{a}$ & $1,332 \mathrm{ef}$ \\
Fusarium tucumaniae CCC 129-11 & $100,00 \mathrm{a}$ & $3,88 \mathrm{~b}$ & $1,955 \mathrm{de}$ \\
Fusarium tucumaniae CCC 134-11 & $100,00 \mathrm{a}$ & $5,00 \mathrm{a}$ & $1,009 \mathrm{f}$ \\
Fusarium tucumaniae CCC 133 11 & $100,00 \mathrm{a}$ & $2,78 \mathrm{~d}$ & $3,020 \mathrm{bc}$ \\
Fusarium virguliforme CCC 101-03 & $100,00 \mathrm{a}$ & $3,32 \mathrm{c}$ & $2,907 \mathrm{c}$ \\
Testigo & $0,00 \mathrm{~b}$ & $1,00 \mathrm{e}$ & $4,509 \mathrm{a}$ \\
\hline CV\% & 0,00 & 4,04 & 18,42 \\
\hline
\end{tabular}

Letras iguales dentro de cada columna indican diferencias no significativas según DMS 5\%.

${ }^{1} \mathrm{CCC}$ : Colección de Cultivos del CEREMIC.

${ }^{2}$ Escala de 1 a 5 donde 1 = planta sana, y 5 = planta muerta.

un rango de 36-75\%, indicando la variación de la incidencia de la enfermedad en las diferentes repeticiones.

Se observaron diferencias estadísticamente significativas entre el peso de 1000 granos de plantas sanas y de plantas enfermas y entre el rendimiento potencial y el real $(p<0,05)$. Los daños promedio fueron de $1514 \mathrm{~kg} / \mathrm{ha}$ (rango $192-3770 \mathrm{~kg} / \mathrm{ha}$ ) lo que significa una pérdida promedio de $22 \%$ (rango 4-46\%). Estas fueron similares a las obtenidas por Freitasi et al. (2004), donde en parcelas de ensayos informaron pérdidas de $1423 \mathrm{~kg} / \mathrm{ha}$. Asimismo, los resultados del presente trabajo fueron semejantes a lo informado por Bird et al. (2010), de la Universidad de Wisconsin, con registros entre 20 y 50\%, y con los determinados en 2009 en Michigan (EEUU), que redujeron en forma significativa el rendimiento (Chilvers, 2011). Otros investigadores como Hartman et al. (1995) observaron una disminución en el rendimiento de hasta $46,2 \%$ en parcelas con alta incidencia (mayor del 50\%). De igual forma, disminuciones de rendimiento de hasta un $80 \%$ se informaron en regiones de un lote y de hasta $40 \%$ en lotes completos en Indiana (Shaner et al., 1998). En Argentina, sobre la variedad A 6445 RG se observó una reducción del 7\% en el peso de 1000 semillas y una disminución de rendimiento por planta del $60 \%$ en un cultivar precoz (Colleto et al., 2008). El 75\% de los lotes relevados en el área de Marcos Juárez, en la campaña 2005/06, presentaron incidencias que variaron entre 1 y $20 \%$ (Distéfano \& Gadbán, 2006), con un 14\% de disminución del rendimiento en un lote comercial y hasta de $41,9 \%$ en parcelas inoculadas versus sin inocular (Lenzi et al., 2007).

Para el peso de 1000 granos, las diferencias entre plantas enfermas versus sanas provenientes del presente ensayo también fueron significativas, del 17,3\%. De esta manera se confirma que el peso es uno de los componentes del rendimiento afectados por esta enfermedad.

Los daños en el rendimiento son variables y dependientes del genotipo, ambiente, condiciones edáficas, fecha de siembra, disponibilidad de $\mathrm{K}$, macroporosidad y $\mathrm{pH}$ del suelo, biotipos presentes del/los patógenos $\mathrm{y}$ posiblemente de otros factores también involucrados
(Estevez de Jensen et al., 2001; Ames et al., 2000; Sanogo et al., 2001; Shaner et al., 1998; Chong et al., 2005). Los resultados de este trabajo confirman que el SMS es una enfermedad que genera significativos daños y que $F$. virguliforme y F. tucumaniae son los agentes causales más frecuentes en la Región Pampeana.

\section{AGRADECIMIENTOS}

Agradecemos a J. Alloatti, R. Wright, B. Ferrari, E. Reinhold por el aporte de muestras de trabajo.

\section{REFERENCIAS}

Ames KA, Ebelhar SE, Barker KL, Pedersen WL (2000) Effect of chloride from potassium chloride fertilizers on Rhizoctonia root and stem rot and sudden death syndrome of soybean. Phytopathology 90:S3.

Aoki T, O'Donnell KL, Scandiani MM (2005) Sudden death syndrome of soybean in South America is caused by four species of Fusarium: Fusarium brasiliense sp. nov., Fusarium cuneirostrum sp. nov., Fusarium tucumaniae and Fusarium virguliforme. Mycoscience 46:162-183.

Aoki T, Scandiani MM, O'Donnell KL (2011). Phenotypic, molecular phylogenetic and pathogenic characterization of Fusarium crassistipitatum sp. nov. Mycoscience. 53:167-186.

Arruda GMT, Miller RNG, Ferreira MASV, Café-Filho AC (2005) Morphological and molecular characterization of the sudden-death syndrome pathogen of soybean in Brazil. Plant Pathology 54:5365 .

Bird G, Bond J, Cianzio S, Conley S, Diers B, Esker P, Leandro L, Malvick, Pedersen P, Yang XB (2010) Soybean sudden death syndrome. Available at: http://www.planthealth.info/pdf_docs/ SDS_Update_2010.pdf. Aceso en: 22/12/2011.

Chilvers M (2011) Soybean sudden death syndrome (SDS) - Fusarium virguliforme. Available at: http://ipmnews.msu. edu/fieldcrop/fieldcrop/tabid/56/articleType/ArticleView/ articleId/3030/Soybean-suddendeath-syndrome-SDS-Fusariumvirguliforme.aspx. Aceso en: 29/07/2010

Chong SK, Hildebranda KK, Luob Y, Myers O, Indorantec SJ, 
M.M. Scandiani et al.

Kazakeviciusand A, Russin J (2005) Mapping soybean sudden death syndrome as related to yield and soil/site properties. Soil and Tillage Research 84:101-107.

Colletto A, Luque A, Salas G, González V, Ploper D, Ruberti D, Biasoli M, Scandiani MM (2008) Identificación de cepas de Fusarium causantes del síndrome de la muerte súbita de la soja en Tucumán y Salta. Avance Agroindustrial 29:26-30.

Covert S, Aoki T, O’Donnell K, Starkey D, Holliday A, Geiser DM, Cheung F, Town A, Strom J, Juba J, Scandiani M, Yang XB (2007) Sexual reproduction in the Sudden Death Syndrome pathogen Fusarium tucumaniae. Fungal Genetics and Biology 44:799-807.

Distéfano S, Gadbán L (2006) Panorama fitopatológico del cultivo de soja en la campaña 2005-2006. Soja actualización 2006. Informe de Actualización Técnica $\mathrm{N}^{\circ} 3$. Marcos Juárez Argentina. INTA EEA.

Estevez de Jensen C, Kurle JE, Percich JA (2001) The effect of chisel and mollboard tillage on dry bean and soybean root rot caused by Fusarium solani f. sp. phaseoli and Rhizoctonia solani in Minnesota. Phytopathology 91:S27.

Freitasi TMQ, Meneghettiii RC, Balardin RS (2004) Dano devido à podridão vermelha da raiz na cultura da soja. Ciência Rural 34:991-996.

Gongora-Canul CC, Leandro LFS (2011) Plant age affects root infection and development of foliar symptoms of soybean sudden death syndrome. Plant Disease 95:242-247.

Hartman GL, Noel GR, Gray LE (1995) Ocurrence of sudden death syndrome in east-central Illinois and associated yield losses. Plant Disease 79:314-318.

Lenzi L, Distéfano S, Salines L (2007) Comportamiento de cultivares de soja frente al Síndrome de la Muerte Repentina con infestación artificial en condiciones de campo. Soja actualización 2007. Informe de actualización técnica $\mathrm{N}^{\circ} 10$. EEA Marcos Juárez INTA.
Li S, Hartman GL, Chen Y (2009) Evaluation of aggressiveness of Fusarium virguliforme isolates that cause soybean sudden death syndrome. Journal of Plant Pathology 91:77-86.

O’Donnell K, Sink S, Scandiani M, Luque A, Colleto A, Biasoli M, Lenzi M, Salas G, González V, Ploper LD, Formento AN, Pioli RN, Aoki T, Yang X B, Sarver BAJ (2010) Soybean sudden death syndrome species diversity within North and South America revealed by multilocus genotyping. Phytopathology 100:58-71.

Ploper LD (1993) Síndrome de la muerte súbita: nueva enfermedad de la soja en el noroeste argentino. Avance Agroindustrial 13:5-9.

Reis EM, Blum MMC, Casa RT, Medeiros CA (1996) Grain losses caused by infection of wheat heads by Gibberella zeae in Southern Brazil, from 1984 to 1994. Summa Phytopathologica 22:134-137.

Rupe JC, Hartman GL (1999) Sudden death syndrome. In: Hartman GL, Sinclair JC, Rupe JC (Eds.) Compendium of Soybean Diseases. $4^{\circ}$ ed. St. Paul MN. APS Press. pp. 37-39.

Sanogo S, Yang XB (2001) Relation of sand content, pH, and potassium and phosporous nutrition to the development of sudden death syndrome in soybean. Canadian Journal of Plant Pathology. 23:174-180

Scandiani MM, Ruberti D, O’Donnell K, Aoki T, Pioli R, Giorda L, Luque A, Biasoli M (2004) Recent outbreak of soybean sudden death syndrome caused by Fusarium virguliforme and Fusarium tucumaniae in Argentina. Plant Disease 88:1044.

Scandiani MM, Ruberti D, Giorda L, Pioli R, Luque A, Bottai H, Ivancovich JJ, Aoki T, O'Donnell K (2011) Comparison of inoculation methods for characterizing relative aggressiveness of two soybean sudden-death syndrome pathogens: Fusarium virguliforme and F. tucumaniae. Tropical Plant Pathology 36:133140.

Shaner GE, Scott DH, Abney DT (1998) Sudden death syndrome in soybeans. West Lafayette IN. Purdue University Cooperative Extension Service.

TPP 512 - Recebido 10 Febrero 2012 - Acceptado 10 Agosto 2012 Editor de Seccion: Luis Rogelio Conci 